\title{
Development of an Integrated Model for Analysis of the Kinetics of Apolipoprotein B in Plasma Very Low Density Lipoproteins, Intermediate Density Lipoproteins, and Low Density Lipoproteins
}

\author{
William F. Beltz, Y. Antero Kesäniemi, Barbara V. Howard, and Scott M. Grundy \\ Veterans Administration Medical Center, San Diego, University of California, San Diego, California 92161; Center for Human \\ Nutrition, Departments of Internal Medicine and Biochemistry, University of Texas Health Science Center, Dallas, Texas 75235, \\ National Institute of Arthritis, Diabetes, Digestive and Kidney Diseases, National Institutes of Health, Phoenix, Arizona 85016; \\ National Cancer Institute, Bethesda, Maryland 20205
}

\begin{abstract}
To quantify more precisely the metabolism of apolipoprotein B (apo B) in human beings, an integrated model was developed for the analysis of the isotope kinetics of apo $B$ in very low density lipoproteins (VLDL), intermediate density lipoproteins (IDL), and low density lipoproteins (LDL). The experimental basis for model development was a series of 30 triple-isotope studies in which patients received autologous ${ }^{131} \mathrm{I}-\mathrm{VLDL},{ }^{125} \mathrm{I}-$ IDL, and $\left[{ }^{3} \mathrm{H}\right] \mathrm{glycerol}$ as a precursor of VLDL triglycerides. The currently proposed model contains the following components: (a) a VLDL delipidation cascade that has a variable number of subcompartments, $(b)$ a slowly catabolized pool of VLDL, (c) an IDL compartment consisting of two closely connected subcompartments, one of which is outside the immediate circulation, and $(d)$ a two-compartment subsystem for LDL. Because mass data indicate that not all VLDL were converted to LDL, the model allows for irreversible removal of apo B from VLDL (or IDL) subsystems. It accounts for apparent "direct" input of LDL by postulating an early, rapidly metabolized compartment of VLDL that is converted directly to IDL. The model appears to be consistent with specific activity curves from the current triple-isotope studies and with present concepts of lipoprotein physiology; it also can be used to quantify pathways of lipoprotein apo B transport in normal and abnormal states.
\end{abstract}

\section{Introduction}

Most cholesterol and triglycerides (TG) ${ }^{1}$ in plasma are transported with lipoproteins containing apolipoprotein B (apo B). Two forms of apo B have been identified in humans (1). One of these, apo B-48, is synthesized in the intestinal mucosa and is associated with chylomicrons. The other, apo B-100, is produced in the liver and is incorporated into TG-rich, very low density lipoproteins (VLDL). In the circulation VLDL undergo lipolytic degradation to smaller lipoproteins-intermediate density lipoproteins (IDL) and low density lipoproteins

\footnotetext{
Dr. Kesäniemi was a visiting scientist on leave from the Second Department of Medicine, University of Helsinki, Helsinki, Finland. 1985.

Received for publication 14 May 1984 and in revised form 1 May
}

1. Abbreviations used in this paper: apo B and E, apolipoproteiti(s) B and E; FCR, fractional catabolic rate; $S_{\mathrm{f}}$, flotation rate; TG, triglyceride; VLDL-B, IDL-B, and LDL-B, very low, intermediate, and low density lipoprotein-apolipoprotein B.

The Journal of Clinical Investigation, Inc.

Volume 76, August 1985, 575-585
(LDL) (2). In this sequence, apo B-100 is retained in the lipoprotein particle. The final product, LDL, is cleared from the circulation mainly by LDL receptors (3). These receptors apparently are specific for apo B-100, although uptake of apo B-containing lipoproteins is facilitated by apolipoprotein $\mathrm{E}$ (apo E) which binds tightly to the receptor $(4,5)$. Thus, not only is circulating LDL removed by LDL receptors, but intermediate lipoproteins, which contain apo $\mathrm{E}$, also seem to be cleared by the same receptors (5); these receptors therefore have been called B-100/E receptors (6).

A number of isotope kinetic studies have been carried out previously to define the metabolism of apo B-containing lipoproteins. The major technique used to study apo B has been to label isolated VLDL or LDL with radioactive iodine (7-27). In a few studies, however, radioactive amino acids have been used as a precursor to apo B (28-30). Early investigations that used radioiodinated lipoproteins employed elementary methods for analyzing isotope kinetic data (7-17, 19-21, 25, 26). Although these investigations have provided useful information about the metabolism of lipoproteins containing apo $\mathrm{B}$, it has become increasingly apparent that a complicated system of metabolic pathways must be taken into consideration to quantify more precisely the kinetics of these lipoproteins. Consequently, kinetic analysis of isotope tracer studies have required increasingly sophisticated approaches, particularly multicompartmental analysis (18, 20-22, 24, 27-30).

The current study was undertaken to develop a more complete model of apo B kinetics to use as a method for evaluating of apo B metabolism. The experimental basis of this investigation was a series of triple-isotope studies in 14 adult Caucasians and 16 Pima Indians. In these studies VLDL was isolated and labeled with ${ }^{131} \mathrm{I}$, and at time of its reinjection, VLDL-TG was labeled endogenously by an intravenous bolus of $\left[{ }^{3} \mathrm{H}\right]$ glycerol. Furthermore, LDL was isolated, labeled with ${ }^{125} \mathrm{I}$, and reinjected. The resulting kinetic curves were compared with previous multicompartmental models, and when the data could not be adequately analyzed by using these models, they were modified to conform with both the present experimental data and our current knowledge of lipoprotein metabolism. The final model was then employed as an analytic tool to quantify various pathways of transport of apo B-containing lipoproteins in normal and abnormal states. The results of these latter analyses are presented in the two following articles $(31,32)$.

\section{Methods}

Patients. The patients for this investigation were studied under metabolic ward conditions either in the Special Diagnostic and Treatment Unit 
of the Veterans Administration Medical Center, San Diego, California, or in the Phoenix Clinical Research Section, National Institutes of Health, Phoenix, Arizona. The characteristics of these patients are described in the following articles $(31,32)$. A total of 30 patients were studied, most of whom had normal plasma lipids. Informed consent was obtained from each patient.

Study design. The protocol was designed to evaluate the kinetics of VLDL-TG and of apo B in three lipoprotein fractions (VLDL, IDL, and LDL). Three radiolabeled materials were injected. VLDL was isolated ultracentrifugally, and its proteins were labeled with ${ }^{131} \mathrm{I}$. LDL also was isolated and labeled with ${ }^{125} \mathrm{I}$. VLDL-TG was labeled by injection of $\left[2{ }^{3} \mathrm{H}\right]$ glycerol as a precursor.

Details of the experimental methods are described in the accompanying articles $(31,32)$. In brief the patients were maintained on a solid food, isocaloric diet for the duration of the study except for receiving a fat-free diet containing $60 \%$ of weight maintenance calories for $36 \mathrm{~h}$ before and for $24 \mathrm{~h}$ (in Phoenix) or $48 \mathrm{~h}$ (in San Diego) after intravenous injection of $\left[{ }^{3} \mathrm{H}\right]$ glycerol and ${ }^{131} \mathrm{I}-\mathrm{VLDL}$. The purpose of this regimen was to eliminate an influx of chylomicrons during the period when VLDL metabolism was assessed. VLDL-TG levels remained in steady state during the study. Blood samples were obtained frequently over the postinjection period. $48 \mathrm{~h}$ after the VLDL injection, the patients received ${ }^{125} \mathrm{I}-\mathrm{LDL}$, and blood samples were obtained frequently during the following $2 \mathrm{~d}$, and then daily in the fasting state for the next 12-19 d. 24-h urine collections were made for each day of the study. Plasma samples were subjected to sequential ultracentrifugation for isolation of VLDL, IDL, and LDL. TG was extracted from VLDL, and its specific activity was determined. Apo B in VLDL, IDL, and LDL was precipitated with tetramethylurea, or with isopropranol as described recently (33), and apo B concentrations and specific activities were determined as described in the companion article $(31,32)$. Study methods at the two locations were carefully standardized to be comparable, and the isoproponal and tetramethylurea methods were shown to yield identical masses for apo B (33).

Analysis of data. The above studies gave specific activity-time curves for VLDL-TG, VLDL-apo B (VLDL-B), IDL-B, and LDL-B, and these specific activity data were analyzed by using a multicompartmental modeling procedure developed by Berman and Weiss (34). Best fits were obtained by using nonlinear regression to minimize the weighted sum of squares (34). The following criteria were used to determine whether a particular model fit the data. First, previously used models were compared to the current data; if they were qualitatively inconsistent, the model was rejected or changed. Second, a model could be rejected for quantitative inconsistency; for example, the model might predict a mass in a pool that was inconsistent with the measured concentration. Third, a model could be rejected for statistical reasons; if the probability of obtaining the computed results with a particular model was less than a certain tolerance, the model was rejected.

A plasma volume of distribution was calculated from the dilution of ${ }^{125} \mathrm{I}-\mathrm{LDL}$ by extrapolation to 0 time of the points obtained during the first 20 min after injection. It was assumed that during this short period no LDL entered the extravascular compartment. The mass of LDL-B in the total plasma compartment was estimated by multiplying the LDL-B concentration by the volume of LDL distribution. The mass of VLDL-B (or IDL-B) in the plasma compartment was assumed to be the product of the VLDL-B (or IDL-B) concentration and the plasma volume computed from the LDL distribution.

\section{Model Development}

\section{Subsystem kinetics}

$V L D L-B$ subsystem. Fig. 1 shows two kinetic curves for VLDL-B resulting from injection of radioiodinated VLDL. Both curves had three components that differed in magnitude but not in basic configuration: an initial flatness (shoulder) for
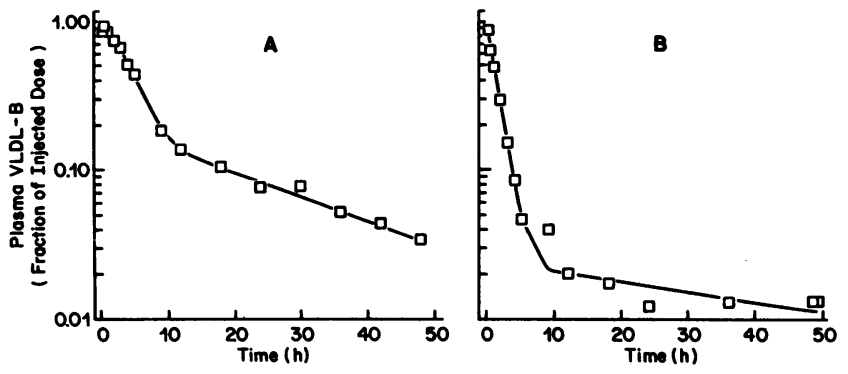

Figure 1. Plasma VLDL-B decay curves from two representative studies. (ㅁ) Measured radioactivities; $(-$ values predicted by the model. Observed and predicted activities are expressed as fractions of the injected ${ }^{131}$ I-VLDL-B.

1-3 h, a phase of more rapid decay to $\sim 10 \mathrm{~h}$, and a slow component (tail) for the remainder of the study. Three models have been used previously for analysis of VLDL-B delay curves of this type. These models and the curves predicted by them are presented in Fig. 2; the latter are plotted with representative experimental data. The first model, which was used in several studies $(9,11,25)$, has a single plasma compartment; it is compatible only with a single-exponential decay. The second model has two compartments, one turning over rapidly and the other more slowly. This model predicts a biphasic curve with a rapid component and a slower tail (35). A third model, proposed by Berman et al. (18), consists of a four-compartment
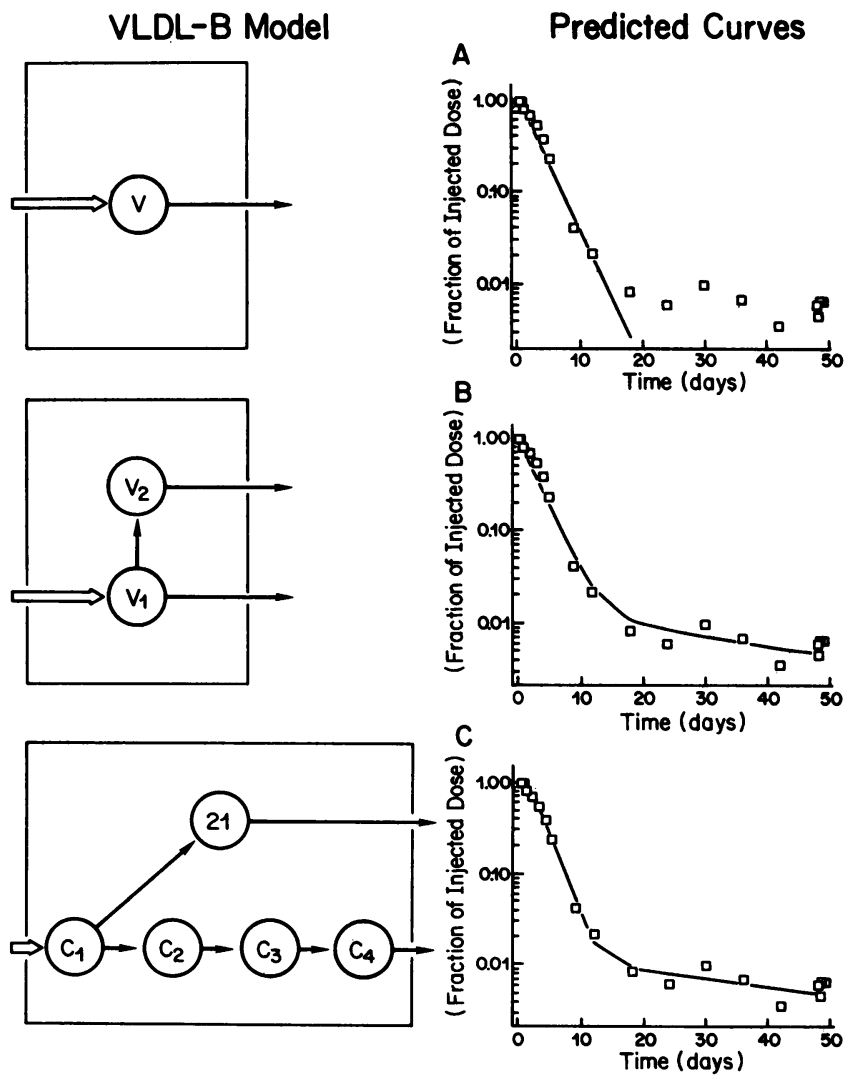

Figure 2. Three proposed models of VLDL-B metabolism and the plasma decay curves predicted by them. All open symbols are from a single turnover study. Observed $(\square)$ and predicted $(-)$ activities are expressed as fractions of the injected VLDL-B dose. The different models and their fits to the observed data are discussed in the text. 
delipidation chain and a single compartment that turns over slowly. The mass of VLDL-B in the chain is equally divided among the pools, and the rate constants are the same for all pools. This chain delipidation pathway accounts for the shoulder before the rapid phase of decay. This model seems superior to the first two because it fits all the components of the curves shown in Fig. 1. As shown by Zech et al. (36) and discussed below, the third model also provides a good description of VLDL-TG kinetics.

Although all of our kinetic curves of VLDL-B had an initial shoulder, the four pools proposed by Berman et al. (18) did not always give the best fit. As shown in Fig. 1, the shoulder can vary considerably in duration and initial slope. By varying the chain length (number of compartments in the delipidation chain), a good fit could be obtained in all cases. Varying the transport rate through the chain was not sufficient to fit the curves, especially those with very "narrow" shoulders. The selection of the best chain length for each study was made as follows. First, the best fit for a four-compartment chain was obtained by adjusting the values of the parameters to minimize the weighted sum of squares. Inspection of the fit to the shoulder suggested whether a longer or shorter chain should be tried. This process was repeated with the new length until a number was found for which either an increase or a decrease in the number of pools resulted in an increase in the sum of squares (i.e., the sum of squares was minimized with respect to the number of compartments). The length of chain that yielded the minimum sum of squares was taken as the best approximation. Occasionally two or more lengths gave comparable fits. In these cases, all competing lengths were used to simultaneously fit the data for VLDL-B and VLDL-TG (see below). The length that gave the minimum sum of squares for both turnover studies was used for further analyses. Table I

Table I. Effect of Delipidation Chain Length on VLDL-B Kinetics*

\begin{tabular}{llllllll}
\hline \multicolumn{7}{c}{ Number of compartments in chain" } \\
\cline { 3 - 8 } Subject no. & & 2 & 3 & 4 & 5 & 6 & 7 \\
\hline \multirow{2}{*}{1} & SS & 1.38 & 1.08 & 1 & 1.02 & & \\
& FCR§ & 0.18 & 0.16 & 0.15 & 0.14 & & \\
5 & SS & 1.04 & 1 & 1.12 & & & \\
& FCR & 0.21 & 0.18 & 0.17 & & & \\
9 & SS & 1.47 & 1.21 & 1.08 & 1.02 & 1 & 1.01 \\
& FCR & 0.15 & 0.14 & 0.13 & 0.12 & 0.12 & 0.11 \\
10 & SS & & 1.01 & 1 & 1.11 & & \\
& FCR & & 0.079 & 0.076 & 0.075 & & \\
11 & SS & 1 & 1.02 & 1.23 & & & \\
& FCR & 0.16 & 0.14 & 0.13 & & & \\
13 & SS & 1.07 & 1 & 1.06 & 1.16 & & \\
& FCR & 0.093 & 0.084 & 0.080 & 0.077 & & \\
\hline
\end{tabular}

* Results are for analysis of the VLDL-B curve only. ‡SS, (sum of weighted squared residuals)/(minimum SS). $\S$ FCR, fractional catabolic rate of VLDL-B in $\mathrm{h}^{-1}$.

"Not all chain lengths were analyzed for each turnover. The best length was taken as that for which either an increase or a decrease in $\mathrm{n}$ caused an increase in SS. shows fractional catabolic rates (FCRs) and weighted sums of squares using chains of differing lengths for each of six representative studies. The sums of squares in Table I are normalized to the minimum found for each study. Note that the best length varied from study to study and that the FCR for a given turnover was dependent on the number of pools used, i.e., increasing the chain length decreased the FCR.

$V L D L-T G$ subsystem. In the current study, $\left[{ }^{3} \mathrm{H}\right]$ glycerol was used as a precursor to VLDL-TG. A characteristic radioactivity-time curve for VLDL-TG is shown in Fig. $3 \mathrm{~A}$ along with specific activity of VLDL-B for the same study. The VLDL-TG curve has several components: an initial delay followed by a rapid upswing in activity, a flatness of the top of the curve, a rapid downswing, and a slow component (tail). Although early workers attempted to estimate the turnover rates of VLDL-TG from the rate of decline of the rapid downswing, it has become apparent that a more complex analysis is required that takes into account all the components of the curve. Thus, Zech et al. (36) proposed a multicompartmental model for a more thorough analysis of VLDL-TG kinetics (Fig. $3 \mathrm{~B}$ ). This model consists of three subsystems: a two-compartment glycerol subsystem, a TG-synthesis subsystem containing both fast- and slow-synthesis pathways, and a

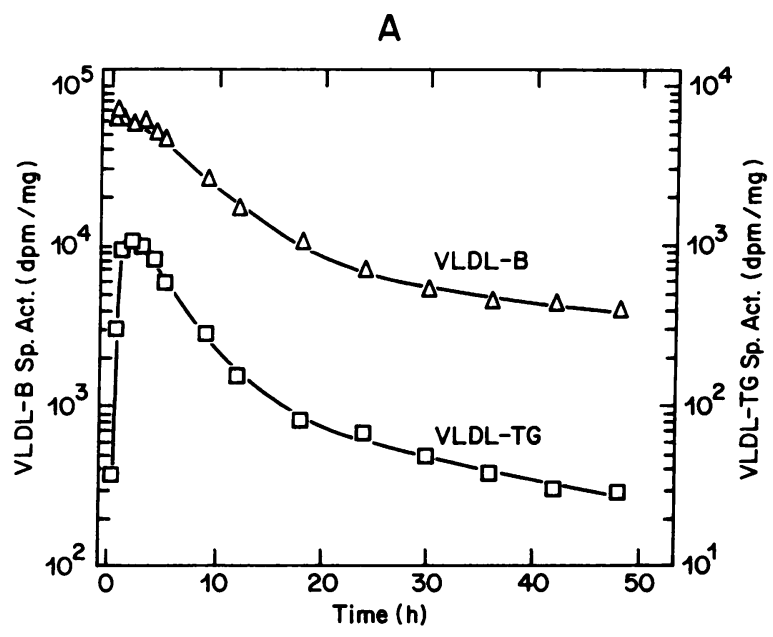

B

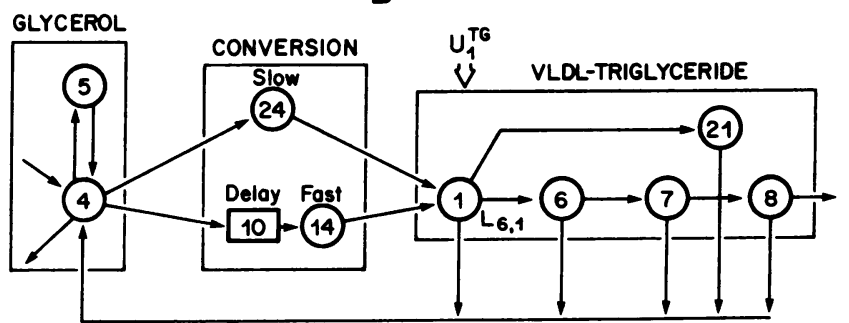

Figure 3. (A) Simultaneous plasma VLDL-B $(\Delta)$ and VLDL-TG (ㅁ) decay curves for a representative turnover study. $(\square, \Delta)$ Measured radioactivities; $(-)$ values predicted by the model. Observed and predicted values are expressed as specific activities. Using the methods as described in references 31 and 32, disintegrations per minute in individual samples ranged from 200 to 10,000 . Masses ranged from 40 to $210 \mu \mathrm{g}$. (B) Model for the kinetics of $\left[{ }^{3} \mathrm{H}\right]$ VLDL-TG proposed by Zech et al. (36). This model was used in the current study, except that the number of compartments in the delipidation chain was not constrained to be four. 
VLDL subsystem, which like the VLDL-B subsystem, contains a rapidly metabolized delipidation chain and a single compartment of more slowly removed VLDL. The glycerol subsystem is required to explain the initial delay before the upswing of radioactivity in plasma VLDL-TG. Published parameters for the glycerol subsystem were used as described in Zech et al. (36). A fast-synthesis pathway can account for the rapid upswing and rapid decay in radioactivity, but a slow pathway of synthesis is needed to account for the major portion of the tail of the curve. The slowly catabolized VLDL pool also contributes to the tail, as shown by the VLDL-B curve. Finally, a delipidation chain is required to explain the flatness at the top of the curve.

When analyzing the kinetics of VLDL-B and VLDL-TG simultaneously, the following constraints were placed on the system parameters. First, the number of pools in the delipidation chain determined for VLDL-B also was used for the VLDLTG model. Second, the residence time (and hence the FCR) of TG and apo B in each compartment of the delipidation chain was the same. This indicates that the residence time in a pool is the same for all components of a particle, even though the different components may take different pathways when they leave the pool. That is, from each compartment of the delipidation chain (compartments 1, 6, 7, and 8 in Fig. 3 $B$ ), VLDL-TG may have one of two fates. First, it may be removed from the particle via lipase activity or exchange with other lipoproteins; lipolysis is thought to exceed exchange greatly (36). Alternately, it may remain with the particle and continue along the chain. Note that the apo B/TG ratios in the compartments of the delipidation chain are not constant, because TG is removed at each step.

The tail of the activity curve of VLDL-B results mainly from the slow conversion of $\left[{ }^{3} \mathrm{H}\right]$ glycerol to TG (36), but a small component of the tail probably is due to the presence of a slowly catabolized compartment, as suggested by the tail on the VLDL-B curve. The turnover of VLDL-TG in this compartment resembles that of $\beta$-VLDL in type 3 hyperlipoproteinemia (18). For this reason, it was assumed that particles in the slowly removed compartment had a composition similar to $\beta$-VLDL. The mass of TG in this pool therefore was estimated using a ratio of apo B/TG of 0.33 , which is the ratio in $\beta$-VLDL (37). The residence time for TG in the slowly catabolized pool also was assumed to be identical to that for apo B which implies that these particles are removed intact from the circulation (18).

$I D L-B$ subsystem. Shapes of specific activity curves for IDL-B $(d 1.006-1.019 \mathrm{~g} / \mathrm{ml})$ showed wide variation among the various studies. Two extremes are presented in Fig. 4. IDL-B curves are plotted along with those for VLDL-B. Immediately after the injection of radioiodinated VLDL-B, a small quantity of label always appeared in the 10-min sample of IDL. After a delay, the subsequent IDL curve rose rapidly, crossed the VLDL-B decay curve, and then decayed. In Figure $4 A$, the IDL-B curve peaks at $\sim 10 \mathrm{~h}$, and the tail remains relatively high at $48 \mathrm{~h}$. In Fig. $4 \mathrm{~B}$, in contrast, the peak occurs at $\sim 4$ $h$ and decays rapidly thereafter with a low tail.

Very few kinetic studies have been reported previously for the IDL-B fraction. Berman et al. (18) used a single-compartment model for the plasma IDL pool. In most of our studies the IDL-B curve was biphasic, suggesting the existence of two plasma pools, one fast and the other slower. However, the amount of apo B transported through the slow IDL-B compartment was found to be very small, and exclusion of this compartment had a trivial impact on estimated transport rates.

LDL-B subsystem. Fig. 5 presents kinetic curves for radioactivity in plasma and urine after injection of radioiodinated LDL. The plasma curve always showed the typical biphasic decay. The urine curve rose rapidly, peaked at $\sim 2 \mathrm{~d}$, and then decayed in parallel with the plasma curve. In some cases, the decay of the urine curve was somewhat faster than the plasma curve. Three models have been suggested for LDL-B kinetics (Fig. 6); these models predict different curves. A two-compartment model (38) generally has been employed for LDL-B kinetics (Fig. $6 \mathrm{~A}$ ). This model hypothesizes a single intravascular pool in equilibrium with an extravascular pool. Input and output are assumed to be from the intravascular compartment. The plasma decay represents the disappearance of a single homogenous pool of LDL in plasma, and the shape of the curve is determined in large part by the exchange process. The model also predicts the urine radioactivity curve shown in Fig. 6 A.

The second model (Fig. $6 \mathrm{~B}$ ) was proposed by Fisher et al. (30). It was developed to explain data from patients with "polydisperse" LDL. Such patients usually have hypertriglyceridemia, and they have been shown to have two species of LDL, one peaking at flotation rate $\left(S_{\mathrm{f}}\right) \mathbf{1 0}$ and another at $S_{\mathrm{f}}$ 4. Fisher et al. (30) labeled LDL-B by injecting the precursor $\left[{ }^{3} \mathrm{H}\right]$ leucine, isolated the two LDL species, and found them to have different kinetic curves. Apparently, when polydisperse LDL is present, two intravascular compartments of LDL are needed. Two intravascular compartments, however, were not required for subjects with single-peak LDL ("monodisperse" LDL). Although the model of Fisher et al. (30) was developed for endogenously labeled LDL, it theoretically could be employed to estimate turnover of exogenously labeled LDL. Fig. $5 B$ shows curves of plasma and urine radioactivity after

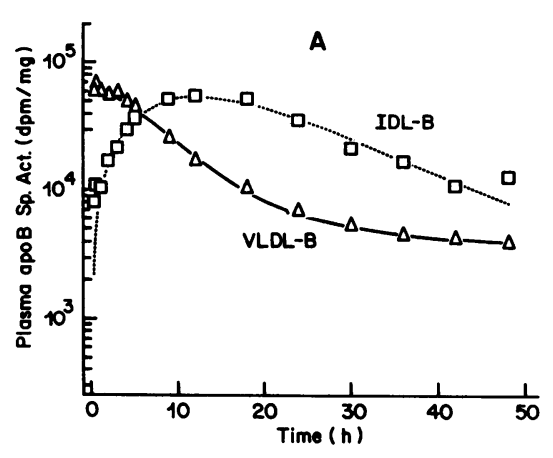

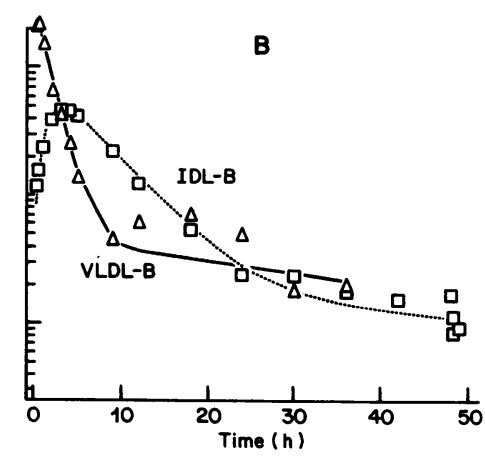

Figure 4. Simultaneous plasma decay curves for VLDL-B $(\Delta)$ and IDL-B ( $\square$ ) for two representative turnover studies. Data obtained from individual VLDL samples were as in Fig. 3. IDL decay ranged from 200 to $16,000 \mathrm{dpm}$, and masses from 15 to 75 $\mu \mathrm{g} .(\Delta, \square)$ Measured radioactivities; $(-)$ values predicted by the model. Observed and predicted values are expressed as specific activities. 


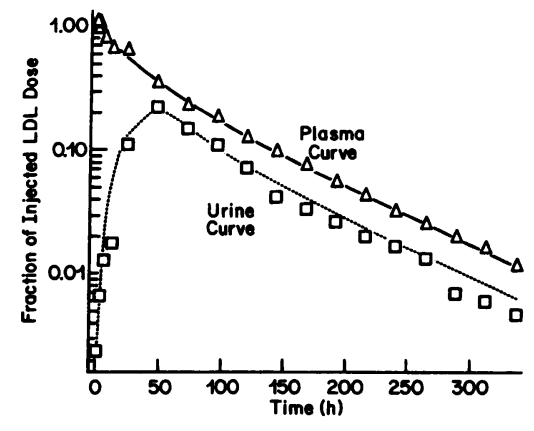

Figure 5. Simultaneous plasma $(\Delta)$ and urine ( $(\square)$ decay curves after injection of labeled LDL-B for a single turnover study. $(\Delta, \square)$ Measured radioactivities; $(-$ ) values predicted by the model. Observed and predicted activities are expressed as fractions of the injected LDL dose.

exogenous labeling that would be predicted from parameter values of Fisher et al. (30). The curves for the $S_{\mathrm{f}} 10$ and $S_{\mathrm{f}} 4$ plasma compartments are also shown. Compartment $S_{\mathrm{f}} 10$, although slower than VLDL, is more rapid than that of compartment $S_{\mathrm{f}} 4$; only the latter was assumed to equilibrate
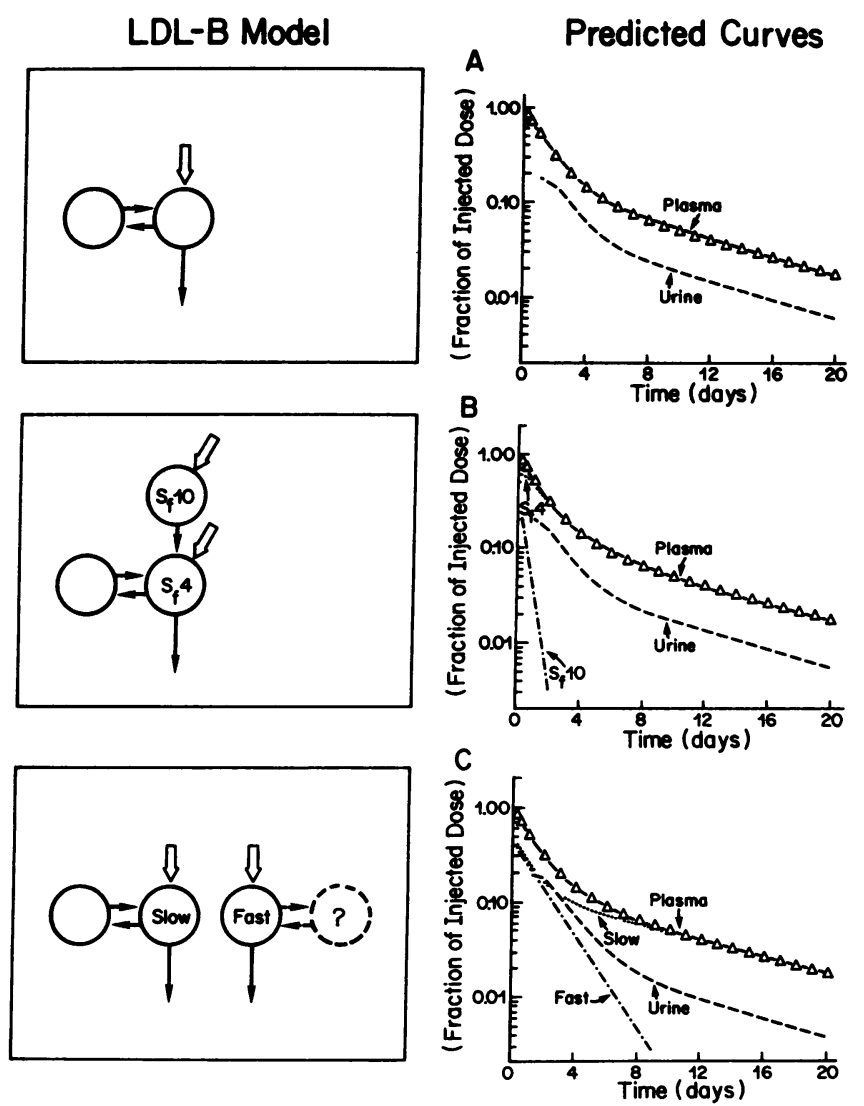

Figure 6. Three proposed models of LDL-B metabolism and the plasma decay curves predicted by them. The open symbols $(\Delta)$, representing observed data, are from the same study in each plot. Data obtained for individual LDL samples ranged from 200 to 1,200 $\mathrm{dpm}$ for ${ }^{131} \mathrm{I}$ and from 200 to $20,000 \mathrm{dpm}$ to ${ }^{125} \mathrm{I}$. Masses were from 600 to $14,000 \mu \mathrm{g}$. Observed and predicted activities are expressed as fractions of the injected dose. The different models are discussed in the text. Also shown are fractions of injected dose for LDL subpopulations (if any) and predicted appearance of activity in urine. with an extravascular pool. According to this model, the plasma curve for exogenously-labeled LDL-B should have a small shoulder. In patients of the current study, most of whom presumably had "monodisperse" LDL, no shoulder could be identified.

The third model was suggested by Goebel et al. (39) (Fig. $6 C$ ). These workers proposed two plasma compartments of LDL with different turnover rates. Although these workers did not postulate an exchange pool for the fast compartment, one could exist. Theoretically, the two plasma pools could be differentiated either by observing a third component in the plasma die-away curve or by a urinary curve from deviating that predicted by the two-pool model. A third component in the plasma curve could not be identified in our patients. Furthermore, most of the observed curves of urine radioactivity fit the two-pool model. When deviations occurred, they were in the later part of the urinary curves when the accuracy of counting was less reliable. For these reasons, the current analyses of LDL-B kinetics were based on the two-pool model (Fig. $6 \mathrm{~A}$ ).

\section{Subsystem integration}

When data from the five labeled species $\left(\left[{ }^{3} \mathrm{H}\right]-\mathrm{VLDL}-\mathrm{TG},{ }^{131} \mathrm{I}-\right.$ VLDL-B, ${ }^{131}$ I-IDL-B, ${ }^{131}$ I-LDL-B, and ${ }^{125}$ I-LDL-B) were integrated, information could be obtained regarding interconversions of apo B-containing lipoproteins and their sites of exit. In addition, the combination of subsystems yielded more information than could be obtained from analysis of individual species. The model integrating the subsystems for VLDL, IDL, and LDL is presented in Fig. 7. This model assumes that most apo B enters plasma as VLDL (compartment $V_{1}$ ). VLDL-B is transported down a delipidation chain of variable length to compartment $V_{\mathrm{n}}$. Apo B in $V_{\mathrm{n}}$ is shown to have one of two fates: $(a)$ conversion to IDL-B (compartment $I_{1}$ ), or $(b)$ irreversible removal from plasma. The slowly catabolized pool, compartment 21 of Berman et al. (18), can be treated as a separate entity. As shown here, apo B can enter and leave this compartment independently of the VLDL chain, but as will

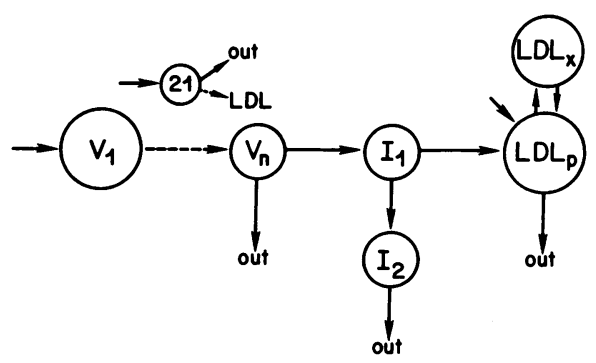

Figure 7. Schematic diagram of the basic components of the multicompartmental model used to analyze data for the current studies $(31,32) . V_{1}$, first compartment of the VLDL delipidation chain; $V_{n}$, last compartment of the VLDL delipidation chain; 21, slowly removed VLDL pool; $I_{1}$, fast IDL pool; $I_{2}$, slow IDL pool; $\mathrm{LDL}_{p}$, LDL plasma pool; $\mathrm{LDL}_{\mathbf{x}}$, LDL extravascular exchange pool. The dashed arrow connecting $V_{1}$ to $V_{\mathrm{n}}$ indicates that other delipidation chain compartments may be present. Predicted plasma VLDL-B activity is calculated as the sum of activities in $V_{1}, \longrightarrow V_{n}$, and 21 . Predicted plasma IDL-B activity is calculated as the sum of activities in $I_{1}$ and $I_{2}$. Predicted plasma LDL activity is the activity in $L L_{p}$. The dashed arrow from compartment 21 to LDL indicates this pathway was required for a small number of subjects. 
be discussed below, essentially identical results are obtained if this pool is derived from the cascade. Most of IDL-B passes through compartment $I_{1}$ to $\mathrm{LDL}$, but a very small portion can be converted to compartment $I_{2}$. Finally, LDL-B can be derived from IDL-B or by "direct" input, that is, independent of the VLDL cascade. Several features of this integrated model must be considered in more detail.

Sources, sizes, and fate of slowly catabolized VLDL pool. The data could not determine whether the slowly metabolized moiety of VLDL was a product of the delipidation chain or of independent synthesis. The origin, however, was of little significance for the overall analysis. Depending on the source, estimates of the pool sizes and transport rates for the slow pool could vary up to $15 \%$, but differences in total transport, chain transport, and FCR of VLDL-B were negligible $(<1 \%)$.

In a few subjects with very high tails for VLDL-B, the amount of ${ }^{131} \mathrm{I}$-apo B entering $\mathrm{LDL}$ appeared to exceed that derived from the fast component of VLDL. This suggested that some of the slowly removed VLDL may have been converted to LDL, and this conversion was allowed. In these patients, allowing the conversion of slow VLDL-B to IDL and LDL also generated a better fit for the peak of the IDL curve. These patients were unusual, however, and the conversion of slowly catabolized VLDL-B to IDL and LDL does not seem appropriate for the general model.

$I D L-B$ transport. In some cases, transport of IDL-B appeared to account for all of the transport of apo B from VLDL to LDL. In other patients, this was not the case (Fig. 8). In this figure, decay curves for VLDL-B, IDL-B, and LDL-B are plotted linearly as fraction of injected dose. The relatively small transport through IDL $(d 1.006-1.019 \mathrm{~g} / \mathrm{dl})$ is illustrated by the small area under the IDL curve compared to the area under the LDL curve. The larger area under the LDL curve is due in part to the longer residence time of $\mathrm{LDL}$, but the discrepancy between the magnitude of IDL and LDL curves is too great to be explained simply by differences in residence time. The IDL curve is too small to allow for all apo B reaching LDL to pass through the measured IDL pool. The

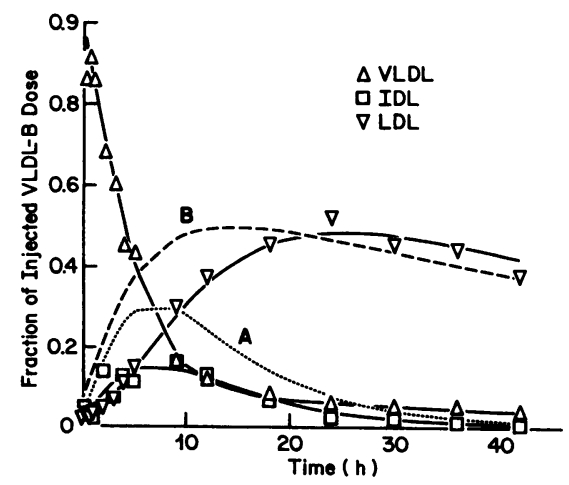

Figure 8. Simultaneous plasma decay curve for VLDL-B ( $\Delta$ ), IDL-B $(\square)$, and LDL-B $(\nabla)$ for a single-turnover study. $(\Delta, \square, \nabla)$ Measured radioactivities; $(\longrightarrow)$ values predicted by the model. Observed and predicted activities are expressed on a linear scale as fractions of the injected VLDL-B dose. The dotted line $(A)$ shows the predicted IDL curve if all VLDL to LDL conversion passes through the measured IDL pool. The dashed line $(B)$ shows the predicted LDL curve if there is no delay in the non-IDL conversion of VLDL to LDL. magnitude of the IDL curve that would be required is illustrated by curve $A$ in Fig. 8 .

In those subjects in whom a significant fraction of VLDL$B$ entered LDL without passing through IDL, a delay between VLDL-B and LDL-B, nevertheless, was observed. This too can be seen in Fig. 8 in which a predicted curve (curve $B$ ) for LDL-B (assuming no delay) is compared to the observed appearance of label in LDL. The displacement of the actual curve to the right is indicative of an unexpected slow conversion of VLDL-B to LDL-B. The data thus suggest that a portion of VLDL-B may leave plasma only to reappear several hours later in LDL. This will be considered further in the Discussion section. Interestingly, the delay between VLDL and LDL was approximately equal to the residence time for IDL-B.

Exit of $V L D L-B$ independent of $L D L$. When the curves for ${ }^{131} \mathrm{I}-\mathrm{LDL}-\mathrm{B}$ and ${ }^{125} \mathrm{I}-\mathrm{LDL}-\mathrm{B}$ were compared, it was noted that VLDL-B usually was not converted quantitatively to LDL (Fig. 9). When the two curves were normalized to fractions of the injected dose, the area under the ${ }^{131}$ I-LDL-B curve (derived from VLDL-B) was less than that of ${ }^{125} \mathrm{I}-\mathrm{LDL}-\mathrm{B}$ (derived from direct injection). This indicates that all ${ }^{131} \mathrm{I}-\mathrm{VLDL}-\mathrm{B}$ did not reach $L D L$. The site of removal of apo $B$ before $L D L$ is unknown. It was assumed that VLDL-B exited from the terminal compartment in the chain $\left(V_{n}\right)$, in that mass data for IDL-B suggested this route. However, some exit from IDL cannot be excluded. A small amount of apo B was also removed via the slow compartment of VLDL.

Length of VLDL delipidation chain. Upon integration of the data it was sometimes possible to refine the estimate of the number of compartments in the VLDL chain. The length of the delipidation chain affects the rate of entry of VLDL-B into IDL and LDL. Thus, by combining the data on the shape of the VLDL shoulder and the delays in conversion to IDL and LDL, the length of the chain could be estimated more accurately than from the shoulder alone.

"Direct" input of LDL-B. When the quantity of VLDL-B converted to LDL-B, as calculated from the ${ }^{131}$ I-LDL-B curve, was compared to LDL-B transport calculated from ${ }^{125} \mathrm{I}-\mathrm{LDL}$ $B$, it was apparent that in many subjects a portion of circulating LDL-B entered plasma without having passed through the

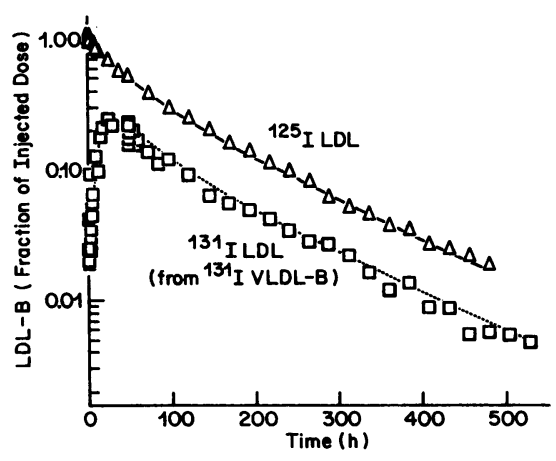

Figure 9. Plasma decay curves of ${ }^{125} \mathrm{I}-\mathrm{LDL}-\mathrm{B}(\Delta)$ and ${ }^{131} \mathrm{I}-\mathrm{LDL}-\mathrm{B}$ (ㅁ) for a single-turnover study. $(\Delta, \square)$ Measured radioactivities; (-, ...) values predicted by the model. The ${ }^{125}$ I-LDL-B decay is after the injection of ${ }^{125} \mathrm{I}-\mathrm{LDL}$. The ${ }^{131} \mathrm{I}-\mathrm{LDL}-\mathrm{B}$ curve is after the injection of ${ }^{131}$ I-VLDL. All data are normalized to the fraction of the injected labeled-apo B dose. If all VLDL were converted to LDL, the areas under these two curves would be equal. 
VLDL-B cascade. This quantity of direct input of LDL-B was variable from person to person, ranging from 0 to $50 \%$. The possible origins of this extra LDL-B will be considered in the Discussion section.

\section{Calculation of transport and production rates}

Production rates of endogenous material were computed by solution of the mass-balance equations for the compartmental model (40). Production rates (apo B or TG) into VLDL-B compartments 21 and $V_{1}$ (Fig. 7) were calculated by constraining the sum of masses in compartments $V_{1}$ through $V_{\mathrm{n}}$ and 21 to equal the measured VLDL mass. The mass of apo B in compartment 21 was estimated by assuming that all VLDL-B was initially labeled with the same specific activity. As described above, the mass of TG in compartment 21 was assumed to be three times the mass of apo $B$. The production rate of apo $B$ into $\mathrm{LDL}$ compartment $\mathrm{LDL}_{\mathrm{p}}$ (Fig. 7) was calculated by constraining the mass in compartment $\mathrm{LDL}_{p}$ to equal the measured LDL apo B mass.

\section{Discussion}

The integrated model shown in Fig. 7 generally was compatible with the kinetic data from the triple-isotope studies of the present investigations. To be a valid representation of apo B metabolism in humans, however, it should conform to knowledge of metabolism obtained in physiologic studies. Numerous physiologic investigations are consistent with the concept that the liver secretes VLDL, and these lipoproteins in turn are catabolized through IDL to LDL. However, in experimental animals, significant amounts of partially degraded VLDL are cleared from the circulation without conversion to LDL. Most of LDL are removed via LDL receptors, and recently, Goldstein et al. (41) demonstrated in experimental animals that VLDL remnants (including IDL) are cleared by the same receptors. Thus, in animal models, the simplest scheme for metabolism of lipoproteins containing apo B-100 is hepatic secretion of VLDL, lipolysis of VLDL to IDL, conversion of IDL or LDL or removal of some IDL by LDL receptors, and removal of most LDL by LDL receptors (41-43).

This scheme (i.e., liver $\rightarrow$ VLDL $\rightarrow$ IDL $\rightarrow$ LDL, with removal of most LDL and some IDL by $L D L$ receptors) almost certainly represents a fundamental pathway, and can serve as the basis for our model, but the current kinetic data are not entirely consistent with its simplicity. Therefore, modifications of this scheme were required by the observed data, and the following discussion examines these key alterations.

$V L D L$ cascade. The above scheme might imply the existence of a single compartment in VLDL; in fact, several previous kinetic studies have employed such a model $(9,11$, 25). Berman et al. (18), however, have shown this to be an oversimplification. Several types of data support a multicompartment VLDL cascade. The flatness at the top of the VLDLTG curve and the shoulder on the VLDL-B curve suggest a cascade $(18,36)$, as does the delayed rise in activity of IDL-B after injection of labeled VLDL-B. Furthermore, the decay curve for VLDL-TG is steeper than for VLDL-B (Fig. 3), implying that TG leaves VLDL before whole particles are cleared. Finally, large VLDL have been shown to be precursors of small VLDL (44-46). The VLDL cascade presumably results from the progressive removal of triglyceride from the particles. This could occur mostly by lipolysis, but possibly to a small extent by transfer of TG to other lipoproteins; the current data cannot distinguish between these two pathways of TG removal from VLDL, but lipolysis almost certainly predominates (36).

The existence of a VLDL cascade thus seems inescapable; still this does not mean that all VLDL must pass through the same number of steps of delipidation. The model shown in Fig. 7 restricts apo $B$ entrance to the beginning of the VLDL chain $\left(V_{1}\right)$ and exit at the termination $\left(V_{n}\right)$. Other models, however, are possible. There could be input or exit at various levels of the cascade; also, the rate of delipidation could decrease at each successive step. We therefore carried out a series of simulations for these different possibilities and compared the simulated results with the model actually used for our analyses. Several statements can be made about these comparisons. First, transport parameters derived from a model with multiple inputs near the beginning of the delipidation chain or with a major input near the end of the chain are very similar to those obtained by using our proposed model that has a changing number of compartments in the delipidation chain. Similarly, parameters derived from a model with a change in rate of delipidation at each step are equivalent to those that use a model with a flexible number of compartments in the chain. The introduction of a variable chain length into the model thus provides a method that basically compensates for multiple inputs or variable lipolytic rates along the cascade, and it gives a better fit to the VLDL curves than a model with a fixed number of compartments. The only situation not accommodated by this model is a significant exit near the beginning of the delipidation chain; in such a case, total VLDL-B transport would be underestimated. Furthermore, removal of apo $B$ from high in the chain could be responsible for the "direct" input of LDL, as discussed below.

"Direct" input of $L D L$. Current data and previous reports $(25,27,42)$ indicate that total transport of LDL-B can exceed estimated amounts of VLDL-B converted to LDL. This phenomenon suggests that apo B mass can enter LDL "directly," i.e., independent of the VLDL cascade. This extra mass of apo B might reach LDL in one of several ways. Direct secretion of IDL with rapid conversion to LDL is one possibility (46). Secretion of LDL-sized particles directly into plasma is another, although if such particles are secreted, they must be modified greatly in plasma before becoming typical circulating LDL (47). Direct input of LDL has been documented in cholesterolfed animals (48-52), but apparently not in normal animals (53-56). It has been suggested from studies in humans that employ transplanchic sampling that direct secretion of LDL may occur (57), but such studies are difficult to interpret. Another mechanism for "direct" input of LDL could be rapid and complete lipolysis of some newly secreted VLDL. A previous study (58) has shown that chylomicron TG undergo lipolysis in a matter of minutes. A similar rapid lipolysis could occur for some VLDL. Such a phenomenon would not be detected in our isotope kinetic data because a pool with rapid entrance and exit from VLDL essentially would be unlabeled. On the basis of studies in normal animals (54-56), we favor this latter mechanism for "direct" input of LDL, although we must stress that our data do not rule out direct hepatic secretion of IDL- or LDL-sized particles in humans.

Role of $I D L$ in apo $B$ transport. A range of different 
patterns of IDL metabolism was found in our subjects. In some patients, IDL-B transport rates were essentially the same as transport of LDL-B; in others, all of apo B transferred from VLDL-B to LDL could not be accounted for by IDL-B kinetics. This discrepancy might have several explanations. First, the "intermediate" lipoprotein might exist predominately in VLDL, as suggested by Fisher et al. $(30,59)$, so that VLDL are transformed directly into LDL without an IDL intermediate. This possibility, however, appears unlikely because it is inconsistent with the observed time delay between VLDL and LDL. Second, the quantity of IDL-B in plasma may have been underestimated because of losses in ultracentrifugation; unfortunately, there is no completely satisfactory way to correct for IDL losses. And third, although Deckelbaum et al. (60) have suggested that all conversion of VLDL to LDL occurs in the "plasma" compartment, this conversion may occur outside the immediately circulating plasma. For instance, Havel (6) has proposed that plasma VLDL remnants attach to LDL (B$100 / E)$ receptors on the surface of liver cells and undergo lipolysis of remaining TG by hepatic TG lipase before being released as LDL. If this hypothesis is true, the transitory IDL phase could exist outside the circulating plasma, i.e., in the space of Disse. In our view, both incomplete recovery of circulating IDL and sequestration of IDL in the conversion pathway to LDL probably contributed to the relatively low transport rates of circulating IDL-B noted in some patients. In that IDL-B was not labeled directly, its metabolism (i.e., "direct" synthesis, direct removal, or exchange with a sequestered or extravascular pool) could not be adequately assessed. Therefore, our proposed structure of the IDL model may be incomplete and thus represents a weakness of the analysis.

Direct removal of $V L D L$ remnants. Although early kinetic data in humans suggested that VLDL-B is transformed quantitatively to LDL-B, there is mounting evidence against this concept. Studies in both animals $(43,61)$ and in humans $(15$, $17,25,62$ ) indicate that VLDL remnants, or IDL, can be removed directly from the circulation. Removal of VLDL remnants appears to be mediated by LDL (B-100/E) receptors. Support for this concept comes from studies in patients with homozygous familial hypercholesterolemia and in the Watanabe heritable hyperlipidemic rabbit; in both, the absence of LDL receptors leads to enhanced input of $\operatorname{LDL}(41,42)$. Varying amounts of VLDL remnants and IDL also might be removed through nonreceptor mechanisms, but whichever the route, the current data bolster the concept of direct removal of VLDL remnants. Although patients with hypertriglyceridemia have been reported previously to have a substantial direct removal of VLDL remnants $(11,44,45)$, data of our companion articles $(31,32)$, as well as data of others $(15,23,25)$, indicate that VLDL-B is not converted quantitatively to LDL, even in normal subjects.

The slow pathway in VLDL. The tail of the VLDL-B curve noted in almost all patients signifies a pool of slowly catabolized VLDL. The size of this pool is greatly increased in type 3 hyperlipoproteinemia (18); this increase results from a structural defect in apo $\mathrm{E}$ that interferes with the clearance of VLDL remnants by the liver. In the absence of an apo E defect, only very small quantities of slowly catabolized VLDL are present. Their origin is unknown. In most patients, it was possible to allow direct exit from this compartment, as seemingly occurs in type 3 hyperlipoproteinemia (18). However, in a few, the pool was somewhat expanded, and it was necessary to transfer its radioactivity to $\mathrm{LDL}$ to account for the full conversion of VLDL-B to LDL. Therefore, the metabolism of slow VLDL$B$ may not be identical in all people.

Model hypothesis. On the basis of our data and current concepts of lipoprotein metabolism, we propose that the scheme presented in Fig. 10 is the best kinetic representation currently available for the metabolism of lipoproteins containing apo B-100. This hypothesis represents an expansion of the model shown in Fig. 7 in that it attempts to reconcile and compartmentalize concepts of lipoprotein metabolism derived from the present data and other physiologic studies. The hypothetical compartments of the model beyond those of Fig. 7 are so designated in Fig. 10. In this model apo B is assumed to enter a hypothetical compartment $\left(V_{0}\right)$, where it can undergo partial lipolysis. Rapid and complete lipolysis in $V_{0}$ would shunt VLDL-B directly to IDL. This pathway could account for observed "direct" input of unlabeled LDL. Because the rate of entrance and exit from $V_{0}$ is relatively high, the mass in this compartment in effect is unlabeled. The model also allows for the possibility of direct secretion of IDL by the liver, a possibility that could not be tested in the present study because labeled IDL was not injected. Our hypothesis assumes that no native $\mathrm{LDL}$ is secreted directly from the liver.

To the extent that lipolysis is incomplete in $V_{0}$, VLDL is degraded to the next step of the cascade $\left(V_{1 \mathrm{a}}\right)$. Here the particle passes through a degradative cascade of variable length to $V_{\mathrm{in}}$. Most apo B leaving $V_{\ln }$ is removed directly from the circulation or is converted to IDL. Apo B exiting $V_{0}$ towards IDL is assumed to be distributed between direct removal and conversion to IDL equally to that leaving $V_{1 \mathrm{n}}$. A small fraction in $V_{1 \mathrm{n}}$ is postulated to enter $V_{2}$, the slowly catabolized compartment of VLDL, although we could not determine whether

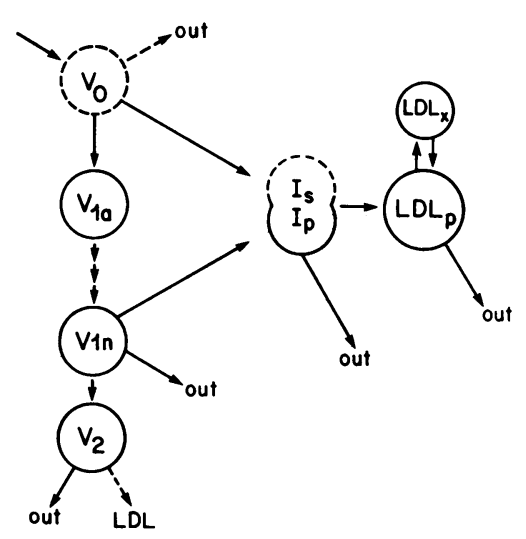

Figure 10. Proposed conceptual model for apo B metabolism. The details of this model are discussed in the text. This model differs from that shown in Fig. 7 in that all apo B is assumed to enter VLDL $\left(V_{0}\right)$. "Direct" input of LDL is considered to represent a direct shunt from $V_{0}$ to IDL $\left(I_{s}\right.$ or $\left.I_{p}\right)$ and LDL. There can be removal of apo B from either VLDL or IDL. Calculation of total apo B transport is higher in this model than that of Fig. 7, because it allows for direct removal of VLDL remnants from $V_{0}$ as well as from $V_{1 \mathrm{n}}$. The dashed arrow from $V_{2}$ to $\mathrm{LDL}$ indicates this pathway was required for a small number of subjects. 
input of $V_{2}$ occurs directly or is through the delipation chain. In large part, apo $\mathrm{B}$ in $V_{2}$ is removed directly from the circulation, although in a few patients it was necessary to divert some apo B in this compartment to LDL.

The IDL pool is hypothesized to consist of one compartment with 2 subcompartments $\left(I_{\mathrm{s}}\right.$ and $\left.I_{\mathrm{p}}\right)$. Subcompartment $I_{\mathrm{p}}$ represents the IDL pool in plasma, whereas subcompartment $I_{\mathrm{s}}$ signifies a sequestered pool of IDL not detected in plasma, whether because of methodologic or true physiologic reasons. The scheme postulates that some apo B leaves IDL directly, but since IDL-B was not labeled independently, it was not possible to detect or measure this pathway. The slowly removed compartment of IDL of Fig. 7 is ignored because of the trivial quantity of apo B transversing it.

The model for LDL postulates two compartments-one intravascular and the other extravascular. Although more compartments in LDL may be required for patients with "polydisperse" LDL (30), both plasma and urine radioactivity of the present study were consistent with the two-pool model.

Although models of greater complexity cannot be excluded from our data, we have attempted to reduce the number of compartments and pathways to as few as possible and still account for the specific activity curves obtained in our studies. Even so, our model is considerably more complicated than the simple scheme for apo B-100 transport described above (i.e., liver $\rightarrow$ VLDL $\rightarrow$ IDL $\rightarrow$ LDL, with removal of most LDL and some IDL by LDL receptors). The following additions beyond this simple scheme were required: $(a)$ a VLDL cascade $\left(V_{0} \rightarrow V_{1 \mathrm{a}} \rightarrow V_{1 \mathrm{n}}\right),(b)$ proximal conversion of VLDL-B to IDL-B $\left(V_{0} \rightarrow I_{\mathrm{p}} / I_{\mathrm{s}}\right),(c)$ a slowly catabolized pool of VLDL $\left(V_{2}\right),(d)$ a sequestered pool of IDL $\left(I_{s}\right)$, and $(e)$ an extravascular exchange pool of LDL (LDL $\mathrm{L}_{\mathrm{x}}$ ). Validation of this model by nonisotopic techniques, unfortunately, has not been possible in humans. However, in the accompanying articles $(31,32)$, data are presented in support of its validity.

Several limitations of the proposed model must be considered. The model undoubtedly is an oversimplication. One weakness probably resides in VLDL. Arbitrary decisions about VLDL model structure were necessary because few experimental data are available on the relationship between input and exit of VLDL-B and VLDL-TG in this complex metabolic pathway. Furthermore, isolation of VLDL in the ultracentrifuge may have altered its metabolic behavior; future studies in which VLDL subfractions are isolated with less drastic methods may provide a more accurate representation of VLDL metabolism. The model for IDL likewise may be incomplete or inaccurate. A sequested pool of IDL outside the circulation but still in continuity with the plasma space has not been demonstrated; the existence of this pool will remain in doubt until methods for quantitative isolation of IDL from plasma are developed. Also, the presence of "direct" input or direct removal pathways for IDL cannot be demonstrated without independent injection of labeled IDL-B. For LDL kinetics, the plasma LDL probably is more heterogeneous than represented. Even in the presence of apparent monodisperse LDL (30), LDL subfractions with different turnover rates may exist. Finally, the liver may directly secrete precursor lipoproteins of the size of IDL and LDL that contribute in part to the "direct" synthesis of LDL. Therefore, further studies may require modification of this model for analysis apo B-100 kinetics.

\section{Acknowledgments}

The authors wish to express their appreciation to the late Dr. Mones Berman who provided inspiration, intellectual stimulation, and facilities at the National Cancer Institute for these investigations.

This research was supported by the Veterans Administration and grants HL-14197 and HL-29252. The research of Dr. Y. Antero Kesäniemi was partly supported by Merrell Dow Pharmaceuticals, Inc., Indianapolis, Indiana. Dr. Scott M. Grundy was a Medical Investigator of the Veterans Administration.

\section{References}

1. Kane, J. P., D. A. Hardman, and H. E. Paulus. 1980. Heterogeneity of apolipoprotein B: isolation of a new species from human chylomicrons. Proc. Natl. Acad. Sci. USA. 77:2465-2469.

2. Havel, R. J., J. L. Goldstein, and M. S. Brown. 1980. Lipoprotein and lipid transport. In Metabolic Control and Disease. 8th edition. P. K. Bondy and L. E. Rosenberg, editors. W. B. Saunders, Philadelphia. 393-494.

3. Goldstein, J. L., and M. S. Brown. 1983. Familial hypercholesterolemia. In The Metabolic Basis of Inherited Disease. 5th edition. J. B. Stanbury, J. B. Wynegarden, D. S. Fredrickson, J. L. Goldstein, and M. S. Brown, editors. McGraw-Hill Book Co., Inc., New York. 672-713.

4. Innerarity, T. L., R. E. Pitas, and R. W. Mahley. 1980. Receptor binding of cholesterol-induced high-density lipoproteins containing predominantly apoprotein $\mathrm{E}$ to cultured fibroblasts with matutions at the low-density lipoprotein receptor locus. Biochemistry. 19:43594365 .

5. Brown, M. S., and J. L. Goldstein. 1983. Lipoprotein receptors in the liver. Control signals for plasma cholesterol traffic. J. Clin. Invest. 72:743-747.

6. Havel, R. J. 1982. Approach to the patient with hyperlipidemia. Med. Clin. N. Am. 66:319-333.

7. Walton, K. W., P. J. Scott, P. W. Dykes, and J. W. L. Davies. 1965. The significance of alterations in serum lipids in thyroid dysfunction. II. Alterations of the metabolism and turnover of ${ }^{131} \mathrm{I}$-lowdensity lipoproteins in hypothyroidism and thyrotoxicosis. Clin. Sci. 29:217-238.

8. Langer, T., W. Strober, and R. I. Levy. 1972. The metabolism of low density lipoprotein in familial type II hyperlipoproteinemia. $J$. Clin. Invest. 51:1528-1536.

9. Bilheimer, D. W., S. Eisenberg, and R. I. Levy. 1972. The metabolism of very low density lipoprotein proteins. I. Preliminary in vitro and in vivo observations. Biochim. Biophys. Acta. 260:212-221.

10. Eisenberg, S., D. W. Bilheimer, R. I. Levy, and F. T. Lindgren. 1973. On the metabolic conversion of human plasma very low density lipoprotein to low density lipoprotein. Biochim. Biophys. Acta. 326: 361-377.

11. Sigurdsson, G., A. Nicoll, and B. Lewis. 1975. Conversion of very low density lipoprotein to low density lipoprotein. A metabolic study of apolipoprotein B kinetics in human subjects. J. Clin. Invest. 56:1481-1490.

12. Sigurdsson, G., A. Nicoll, and B. Lewis. 1976. Metabolism of very low density lipoproteins in hyperlipidemia: studies of apolipoprotein B kinetics in man. Eur. J. Clin. Invest. 6:167-177.

13. Packard, C. J., J. L. H. C. Third, J. Shepherd, A. R. Lorimer, H. G. Morgan, and T. D. V. Lawrie. 1976. Low density lipoprotein metabolism in a family of familial hypercholesterolemic patients. Metab. Clin. Exp. 25:995-1006.

14. Thompson, G. R., and N. B. Myant. 1976. Low density lipoprotein turnover in familial hypercholesterolemia after plasma exchange. Atherosclerosis. 23:371-377. 
15. Sigurdsson, G., A. Nicoll, and B. Lewis. 1977. Turnover of apolipoprotein-B in two subjects with familial hypobetalipoproteinemia. Metab. Clin. Exp. 26:25-31.

16. Nicoll, A., G. Sigurdsson, A. Marsh, and B. Lewis. 1977. Intravenous fat tolerance. Correlation with very low density lipoprotein apoprotein B kinetics in man. Atherosclerosis. 26:17-22.

17. Reardon, M. F., N. H. Fidge, and P. J. Nestel. 1978. Catabolism of very low density lipoprotein B apoprotein in man. J. Clin. Invest. 61:850-860.

18. Berman, M., M. Hall, R. I. Levy, S. Eisenberg, D. W. Bilheimer, R. D. Phair, and R. H. Goebel. 1978. Metabolism of apoB and apoC lipoproteins in man: kinetic studies in normal and hyperlipoproteinemic subjects. J. Lipid Res. 19:38-56.

19. Nestel, P. J., M. Reardon, and N. H. Fidge. 1979. Sucroseinduced changes in VLDL- and LDL-B apoprotein removal rates. Metab. Clin. Exp. 28:531-535.

20. Bilheimer, D. W., N. J. Stone, and S. M. Grundy. 1979. Metabolic studies in familial hypercholesterolemia. Evidence for a gene-dosage effect in vivo. J. Clin. Invest. 64:524-533.

21. Calvert, G. D., and H. M. James. 1979. Low-density lipoprotein turnover studies in man. Evaluation of the integrated rate equations method, use of a whole-body radioactivity counter, and the problem of partial denaturation. Clin. Sci. 56:71-76.

22. Shepherd, J., C. J. Packard, S. M. Grundy, D. Yeshurun, A. M. Gotto, and O. D. Taunton. 1980. Effects of saturated and polyunsaturated fat diets on the chemical composition and metabolism of low density lipoproteins in man. J. Lipid Res. 21:91-99.

23. Packard, C. J., J. Shepherd, S. Joerns, A. M. Gotto, and O. D. Taunton. 1980. Apolipoprotein B metabolism in normal, type IV, and type V hyperlipoproteinemic subjects. Metab. Clin. Exp. 29:213-221.

24. Melish, J., N. A. Le, H. Ginsberg, D. Steinberg, and W. V. Brown. 1980. Dissociation of apoprotein B and triglyceride production in very-low-density lipoproteins. Am. J. Physiol. 239:E354-E362.

25. Janus, E. D., A. Nicoll, R. Wootton, P. R. Turner, P. J. Magill, and B. Lewis. 1980. Quantitative studies of very low density lipoprotein: conversion to low density lipoprotein in normal controls and primary hyperlipidemic states and the role of direct secretion of low density lipoprotein in heterozygous familial hypercholesterolemia. Eur. J. Clin. Invest. 10:149-159.

26. Janus, E. D., A. M. Nicoll, P. R. Turner, P. Magill, and B. Lewis. 1980. Kinetic bases of the primary hyperlipoproteinemias: studies of apolipoprotein B turnover in genetically defined subjects. Eur. J. Clin. Invest. 10:161-172.

27. Ginsberg, H. N., N. A. Le, J. Melish, D. Steinberg, and W. V. Brown: 1981. Effect of a high carbohydrate diet on apoprotein-B catabolism in man. Metab. Clin. Exp. 30:347-353.

28. Eaton, R. P., and D. M. Kipnis. 1972. Incorporation of $\mathrm{SE}^{75}$ selenomethionine into a protein component of plasma very-low-density lipoprotein in man. Diabetes. 21:744-753.

29. Phair, R. D., M. G. Hammond, J. A. Bowden, M. Freid, W. R. Fisher, and M. Berman. 1975. Preliminary model for human lipoprotein metabolism in hyperlipoproteinemia. Fed. Proc. 34:22632270.

30. Fisher, W. R., L. A. Zech, P. Bardalaye, G. Warmke, and M. Berman. 1980. The metabolism of apolipoprotein B in subjects with hypertriglyceridemia and polydisperse LDL. J. Lipid Res. 21:760-774.

31. Kesäniemi, Y. A., W. F. Beltz, and S. M. Grundy. 1985. Comparisons of metabolism of apolipoprotein B in normal subjects, obese patients, and patients with coronary heart disease. J. Clin. Invest. 76:586-595.

32. Egusa, G., D. W. Brady, S. M. Grundy, and B. V. Howard. 1985. The influence of obesity on the metabolism of apolipoprotein B in humans. J. Clin. Invest. 76:596-603.

33. Egusa, G., D. W. Brady, S. M. Grundy, and B. V. Howard. 1983. Isopropanol precipitation method for the determination of apolipoprotein B specific activity and plasma concentrations during metabolic studies of very low density lipoprotein and low density lipoprotein apolipoprotein B. J. Lipid Res. 21:1261-1267.

34. Berman, M., and M. F. Weiss. 1978. "SAAM Manual." Department of Health, Education and Welfare Publ. No. (NIH) 78180. U. S. Government Printing Office, Washington, DC.

35. Fisher, W. R. 1982. Apoprotein B kinetics in man: concept and questions. M. Berman, S. M. Grundy, and B. V. Howard, editors. In Lipoprotein kinetics in modeling. Academic Press, Inc., New York. 43-68.

36. Zech, L. A., S. M. Grundy, D. Steinberg, and M. Berman. 1979. Kinetic model for production and metabolism of very low density lipoprotein triglycerides. Evidence for a slow production pathway and results for normolipidemic subjects. J. Clin. Invest. 63:1262-1273.

37. Pagnan, A., R. J. Havel, J. P. Kane, and L. Kotite. 1977. Characterization of human very low density lipoproteins containing two electrophoretic populations: double pre-beta lipoproteinemia and primary dysbetalipoproteinemia. J. Lipid Res. 18:613-622.

38. Matthew, C. M. E. 1957. The theory of tracer experiments with ${ }^{131}$ I-labeled plasma proteins. Phys. Med. Biol. 2:36-53.

39. Goebel, R., M. Garnick, and M. Berman. 1976. A new model for low density apolipoprotein kinetics: evidence for two labeled moieties. Circulation 54(Suppl. II):4.

40. Berman, M. 1982. Kinetic analysis and modeling: theory and applications to lipoproteins. In Lipoprotein Kinetics and Modeling. M. Berman, S. M. Grundy, and B. V. Howard, editors. Academic Press, Inc., New York. 3-36.

41. Goldstein, J. L., T. Kita, and M. S. Brown. 1983. Defective lipoprotein receptors and atherosclerosis. N. Engl. J. Med. 309:288296.

42. Bilheimer, D. W., Y. Watanabe, and T. Kita. 1982. Impaired receptor-mediated catabolism of low density lipoprotein in the WHHL rabbit, an animal model of familial hypercholesterolemia. Proc. Natl. Acad. Sci. USA. 79:3305-3309.

43. Kita, T., M. S. Brown, D. W. Bilheimer, and J. L. Goldstein. 1982. Delayed clearance of very low density and intermediate density lipoproteins with enhanced conversion to low density lipoprotein in WHHL rabbits. Proc. Natl. Acad. Sci. USA. 79:5693-5697.

44. Streja, D., M. A. Kallai, and G. Steiner. 1977. The metabolic heterogeneity of human very low density lipoprotein triglycerides. Metab. Clin. Exp. 26:1333-1344.

45. Reardon, M. F., M. E. Poapst, and G. Steiner. 1982. The independent synthesis of intermediate density lipoproteins in type III hyperlipoproteinemia. Metab. Clin. Exp. 31:421-427.

46. Fidge, N. H., and P. Poulis. 1978. Metabolic heterogeneity in formation of low density lipoprotein from very low density lipoprotein in rat: evidence for independent production of a low density lipoprotein subfraction. J. Lipid Res. 19:342-349.

47. Glomset, J. A., and K. R. Norum. 1973. The metabolic role of lecithin: cholesterol acyltransferase: perspectives from pathology. Adv. Lipid Res. 11:1-65.

48. Illingworth, D. R. 1975. Metabolism of lipoproteins in nonhuman primates. Studies on the origin of low density lipoprotein apoprotein in the plasma of the squirrel monkey. Biochim. Biophys. Acta. 388:38-51.

49. Nakaya, N., B. H. Chung, and O. D. Taunton. 1977. Synthesis of plasma lipoproteins by the isolated perfused liver from the fasted and fed pig. J. Biol. Chem. 252:5258-5261.

50. Nakaya, N., B. H. Chung, J. R. Patsch, and O. D. Taunton. 1977. Synthesis and release of low density lipoproteins by the isolated perfused pig liver. J. Biol. Chem. 252:7530-7533.

51. Noel, S. P., L. Wong, P. J. Dolphin, L. Dory, and D. Rubenstein. 1979. Secretion of cholesterol-rich lipoproteins by perfused livers of hypercholesterolemic rats. J. Clin. Invest. 64:674-683.

52. Johnson, F. L., R. W. St. Clair, and L. L. Rudel. 1983. Studies on the production of low density lipoproteins by perfused livers from 
nonhuman primates. Effect of dietary cholesterol. J. Clin. Invest. 72: 221-236.

53. Hamilton, R. L., M. C. Williams, C. J. Fielding, and R. J. Havel. 1976. Discoidal bilayer structure of nascent high density lipoproteins from perfused rat liver. J. Clin. Invest. 58:667-680.

54. Guo, L. S., R. L. Hamilton, R. Ostwald, and R. J. Havel. 1982. Secretion of nascent lipoproteins and apolipoproteins by perfused livers of normal and cholesterol-fed guinea pigs. J. Lipid Res. 43-555.

55. Hornick, C. A., T. Kita, R. L. Hamilton, J. P. Kane, and R. J. Havel. 1983. Secretion of lipoproteins from the liver of normal and watanabe heritable hyperlipidemic rabbits. Proc. Natl. Acad. Sci. USA. 80:6096-6100.

56. Marsh, J. B. 1976. Apoproteins of the lipoproteins in a nonrecirculating perfusate of rat liver. J. Lipid Res. 17:85-90.

57. Turner, R. R., N. E. Miller, C. Cortese, W. Hazzard, J. Coltart, and B. Lewis. 1981. Splanchnic metabolism of plasma apoprotein B. J. Clin. Invest. 67:1678-85.
58. Grundy, S. M., and H. Y. I. Mok. 1976. Chylomicron clearance in normal and hyperlipidemic man. Metab. Clin. Exp. 25:1225-1239.

59. Fisher, W. R. 1970. The characterization and occurrence of an S 20 serum lipoprotein. J. Biol. Chem. 245:877-884.

60. Deckelbaum, R. J., S. Eisenberg, Y. Oschry, E. Butbul, I. Sharon, and T. Olivecrona. 198. Reversible modification of human plasma. Low density lipoproteins toward triglyceride-rich precursors. J. Biol. Chem. 257:6509-6517.

61. Mjos, O. D., O. Faergeman, R. L. Hamilton, and R. J. Havel. 1975. Characterization of remnants produced during the metabolism of triglyceride-rich lipoproteins of blood plasma and intestinal lymph in the rat. J. Clin. Invest. 56:603-615.

62. Sniderman, A., D. Thomas, B. Marpole, and D. Teng. 1978. Low density lipoprotein: a metabolic pathway for return of cholesterol to the splanchnic bed. J. Clin. Invest. 61:867-873. 